\title{
Coexistencia multicultural a nivel local: oenegés migrantes e integración de los nikkeijin en el este de Japón
}

DOI: $10.32870 /$ mycp.v2i5.411

Marcela Méndez Vázquez ${ }^{1}$

\section{Resumen}

El importante incremento en Japón de residentes de ascendencia japonesa (nikkeijin) desde 1990 en localidades donde conviven con los nacionales y otros grupos étnicos, ha motivado medidas destinadas a atender las demandas planteadas por su presencia. Siguiendo directrices del Estado agrupadas bajo los términos "coexistencia multicultural", los gobiernos locales han ensayado diversas estrategias para atenderlas.

Basado en un trabajo de campo que tuvo lugar desde agosto de 2007, hasta agosto de 2008, este artículo acomete dos tareas: primero, compara cómo nikkeijin que viven en Kanto y en Tokai se redefinen como agentes interactuando con las organizaciones locales y los municipios, procurando ciudadanía en el ámbito local en el marco de la coexistencia multicultural o tabunka kyôsei. En segundo lugar, propone que el análisis de las políticas hacia los extranjeros y la ciudadanía debe dar cuenta de lo local y lo regional, además de ser sensible al género y a la historia.

Palabras clave: nikkeijin, coexistencia multicultural, tabunka kyôsei, ciudadanía, género.

Artículo recibido el 30 de marzo de 2013 y dictaminado el 17 de mayo de 2013.

1. Doctora en Estudios de Asia y África por El Colegio de México, A. C. Profesora huésped en el Departamento de Estudios del Pacífico, Cucsh de la Universidad de Guadalajara. 


\section{Abstract}

Since the 1990s, Japan has received immigrant workers of Japanese ancestry (nikkeijin) that now reside together with nationals and other ethnics groups. To cope with the impact of their demands, local governments have been implementing measures called "multicultural coexistence". Based upon long term fieldwork (2007-2008), this paper addresses two tasks: firstly, compares how nikkeijin living in Kanto and Tokai redefined themselves as agents interacting with the local autonomies and organizations, seeking integration and finding citizenship al the local level within the multicultural coexistence guidelines.

Secondly, it holds that the analysis of policies towards foreigners in Japan must account for the local and the regional, besides taking into account gender and history, risking misconstructions of not doing so.

Keywords: nikkeijin, tabunka kyôsei, multicultural coexistence, citizenship, gender.

\section{Introducción}

Al manifestarse la crisis financiera mundial de septiembre de 2008 en Japón, la respuesta política del Gobierno para los extranjeros llegó en enero de 2009, cuando se hicieron públicas normativas específicamente destinadas a paliar las consecuencias de los despidos sufridos por los nikkeijin. ${ }^{2}$ Ese mes se abrió una oficina con el propósito de elaborar iniciativas para los residentes foráneos bajo la esfera de la oficina del gabinete del primer ministro, una decisión sin precedentes por su potencial para promover la integración social. ${ }^{3}$ De las medidas propuestas, como la promoción de la enseñanza del idioma japonés

2. Los nombres propios en japonés se han colocado en el orden usual en español: nombre y apellido. En cuanto a los documentos, se cita primero la versión consultada y luego mi traducción. Se aclara si se utiliza la traducción provisional al español por el Estado japonés. Para los cargos y organismos se utiliza la traducción oficial del Estado japonés en español, si la hubiere.

3. La Oficina para la Promoción de Medidas para Extranjeros Residentes (Teijû gaikokujin shisaku suishin shitsu) se estableció el 9 de enero de 2009, para diseñar políticas en colaboración con los ministerios y agencias pertinentes y promover su implementación por los gobiernos locales. Publicaron sus directrices el 30 de enero de ese año, las cuales cubren las áreas de: educación, empleo, vivienda, apoyo al regreso de nikkeijin y el suministro de información. Las mismas se sistematizan en su primer documento Teijû gaikokujin shien ni kansuru tômen no taisaku ni tsuite (De de las medidas urgentes de apoyo a los residentes extranjeros (mi traducción). Disponible en http:// www8.cao.go.jp/teiju/taisaku/pdf/taisaku_z.pdf (Consultado el $1^{\circ}$ de enero de 2013). 
o la capacitación técnica, ${ }^{4}$ la asignación extraordinaria para apoyar el retorno de los nikkeijin a sus países de origen con la condición de que no regresaran, fue una de las más publicitadas en los medios. ${ }^{5}$

A partir de la crisis se generaron algunas posibilidades antes no disponibles para quienes permanecieron en el país. A este respecto, son necesarias tanto la evaluación de la efectividad de las medidas implementadas en el momento álgido de la crisis global, cuando los trabajadores nikkeijin y el resto de la fuerza laboral, junto con ellos, fueron afectados por el desempleo; como la evaluación de la situación de los trabajadores extranjeros en vísperas del gran terremoto del este de Japón del 11 de marzo de 2011, ${ }^{6}$ cuando se vislumbraban signos de mejora (Ando y Kimura, 2012: 261-287), problemáticas que se encuentran fuera del alcance de este trabajo.

4. El Gobierno destinó mil millones de yenes (10’090,000 USD al 31/3/2009) para programas de idioma y capacitación técnica. Asimismo, en abril de 2009 el Ministerio de Salud, Trabajo y Bienestar comenzó a ofrecer una asignación única de apoyo de 300,000 yenes a cada nikkeijin adulto que partiera y 200,000 por cada dependiente, siempre que accedieran a no regresar a Japón. Esta condición se modificó a posteriori, cuando se redujo a una limitación de "no retorno por tres años". El 27 de marzo se creó el Consejo para la Promoción de Medidas para Residentes Extranjeros [Nikkei teijû gaikokujin shisaku suishin kaigi], comprendiendo un ministro de Estado, viceministros, y a otros altos funcionarios de burós y agencias para coordinar las políticas. Está disponible en http:// www.mhlw.go.jp/houdou/2009/03/h0331-10.html

http://www8.cao.go.jp/teiju/kaigi/kaisai.html (Consultado el $1^{\circ}$ de enero de 2013).

5. El programa administrado por el Ministerio de Trabajo terminó el 31 de marzo de 2010. Véase: "Sobre la terminación de la ayuda para el retorno de los desempleados nikkei" [Nikkei rishokusha ni taisuru kikoku shien jigyô no shûryô ni tsuite], http://www.mhlw.go.jp/stf/houdou/2r98520000003rnz.html Y también el documento anexo: "Resumen del plan de ayuda para el retorno de los desempleados nikkei" [Nikkei rishokusha ni taisuru kikoku shien jigyô no gaiyô]. Disponible en http://www.mhlw.go.jp/stf/houdou/2r98520000003rnz-img/2r98520000003rph.pdf (Consultados el 25 diciembre de 2012).

6. El 11 de marzo de 2011 a las 14:56 Jst, la región japonesa de Tohoku sufrió un sismo de magnitud 9 MM, seguido por un tsunami y una posterior emergencia nuclear en la planta Fukushima Daiichi que provocó la fusión de tres reactores nucleares. El desastre causó 20,000 víctimas y el daño en la región fue evaluado en unos $¥ 20$ billones (USD 220 mil millones).

"Higashi Nihon Daishinsai kara ikkagetsu. Ikiru imi wo saburareta sanjûnichikan. Wareware ha nani wo towareteiru ka", Diamond Online, 11 de abril de 2011. http://diamond.jp/articles/-/11827 (Consultado el $1^{\circ}$ de febrero de 2013).

"Ni, san gô ki mo merutodaun no kanôsei... Tôden mitomeru", Yomiuri Shimbun, 14 de mayo de 2011, edición en línea. http://www.yomiuri.co.jp/science/news/20110514-oyt1t00824.htm

(Consultado el $1^{\circ}$ de febrero de 2013).

“Three nuclear reactors melted down after quake, Japan confirms", cNN World, 6 de junio de 2011, edición en línea.

http://articles.cnn.com/2011-06-06/world/japan.nuclear.meltdown_1_nuclear-reactors-fuelrods-tokyo-electric-power? (Consultado el 30 de octubre de 2012). 
El presente artículo se centra en la época previa a la crisis financiera de 2008, analizando la acción colectiva de organizaciones de nikkeijin en procura de ciudadanía e integración de sus comunidades en Japón, presentando el caso de dos entidades creadas por residentes peruanos. ${ }^{7}$

\section{Coexistencia multicultural y el Japón monoétnico}

A partir de 2005, el Gobierno central ha enmarcado medidas relativas a los extranjeros dentro del término "coexistencia multicultural" (tabunka kyôsei). En el discurso oficial el mismo remite a la convivencia (coexistencia) de japoneses y no-japoneses ${ }^{8}$ y lentamente se ha impuesto al término "internacionalización" (kokusaika), empleado con anterioridad. Lejos de ser ahistórico como se presenta en los textos oficiales, debe su emergencia a movimientos en la sociedad civil liderados por distintas minorías: los descendientes de los burakumin, ${ }^{9}$ los ainu y los okinawenses (Siddle, 1996), y los ex súbditos imperiales coreanos y taiwaneses. Inclusive los activistas burakumin ya utilizaban esa expresión en los años setenta (Hanazaki, 2002). Si la coexistencia multicultural aparece en el discurso oficial, ¿qué ha ocurrido con la idea de un Japón homogéneo o monoétnico?, ¿cómo se integra a los extranjeros recientemente llegados? Observemos brevemente el desarrollo histórico de la identidad nacional y la incorporación de la otredad.

Los Estados-nación se basan en una ideología que sostiene que sus fronteras políticas tienen que colindar con fronteras culturales. Las identidades nacionales, como otras identidades étnicas, se forman en relación con los otros, otras naciones u otras gentes que no forman parte de la nación. Estos otros con culturas diferentes son divididos en miembros del grupo y no

7. El número de residentes brasileños registrados a finales de 2008 era de 312,582 , mientras que el número de peruanos era de 59,723. A partir del otoño de 2008, el número de salidas de extranjeros superó a las entradas. Así, el número de brasileños registrados a finales de 2009 disminuyó en unos 45,126 (14.4\%), llegando a un total de 267,456, y el de peruanos disminuyó en 2,259 (3.8\%) totalizando unos 57,464. http://www8.cao.go.jp/teiju-portal/eng/taisaku/index.html\#1 (Consultados el 25 diciembre de 2012).

8. El término aparece por primera vez en un documento del gobierno central en 2005, unas directrices del Ministerio de Asuntos Internos y Comunicaciones, cuyo título es: Tabunka kyôsei shakai wo mezashita torikumi (Medidas para una sociedad de coexistencia multicultural).

http://www.clair.or.jp/j/forum/forum/articles/sp/207_2/index.html (Consultado el 30 de agosto de 2012).

9. Personas que realizaban oficios considerados contaminantes (carniceros, enterradores, curtidores, etc.) y cuya emancipación se decretó a comienzos de la era Meiji. 
miembros, y en esa operación se les concede ciudadanía (derechos) (Eriksen, 2002: 110-113).

La expansión territorial de Japón a partir de la Restauración Meiji (1868) estuvo acompañada de la expansión de la ciudadanía, la que contempló la incorporación de los "otros" internos en primera instancia. Desde el centro, se incorporaron los ainu moshir - a través de la ocupación efectiva de Hokkaido en 1869- y luego los okinawenses — con la terminación del Reino de Ryukyu como prefectura en 1879 , completando la colonización interna-. La colonización de territorios de ultramar incorporó en principio a Taiwán (cedido por China luego de su derrota en la Primera Guerra Sino-japonesa, 1895) y luego a Corea (anexada en 1910) (Oguma, 1998). Los pueblos colonizados eran considerados súbditos del emperador Meiji, por lo que debían aprender japonés y mostrarle respeto. Así, los coreanos - y taiwanesas-, súbditos de ultramar (gaichi), nunca fueron vistos de la misma manera que los súbditos de naichi (del interior). ${ }^{10}$ Si bien el gobierno imperial enfatizó una identidad racial común a los asiáticos, también estableció una identidad nacional japonesa diferenciada en una jerarquía entre diferentes etnicidades (Doak 2007). Los de gaichi fueron tratados como ciudadanos de segunda categoría, no sólo en términos de su ciudadanía, sino también de su identidad cultural. La jerarquía étnica también se reprodujo en el discurso de la Esfera de Co-prosperidad del Gran Este Asiático (Tai, 2004). Pero las jerarquías no culminaron con el imperio, han persistido hasta la actualidad y permean las políticas migratorias y la concesión de ciudadanía (Shipper, 2008; Chung, 2010; Méndez Vázquez, 2011).

Si la diversidad interna fue incorporada en el proceso constitutivo de Japón como Estado-nación moderno, y luego ampliada con la diversidad de ultramar durante la expansión imperial, ¿de dónde surge la idea del Japón homogéneo? El argumento de Oguma (2002) es que la imagen de Japón como una sociedad homogénea no es el producto del discurso previo a la Segunda Guerra Mundial, sino de la posguerra. El discurso anterior a la guerra sobre la identidad japonesa está vinculado al imperialismo, racismo y nacionalismo para justificar la dominación sobre los países asiáticos circundantes, pero a la vez proponía un imperio con variedad racial y étnica — por ejemplo, el

10. Además de las etnias incorporadas a naichi, al comienzo de Meiji también llegaron extranjeros contratados (oyatoi gaikokujin) por miles, para asesorar al Estado y al sector privado en la modernización (Jones, 1975). 
El caso de los nikkeijin es paradójico, pues a través de la modificación de 1990 a la Ley de Control a la Inmigración, la identidad nikkei ha sido empleada por el Estado para justificar convocarlos como similares étnicos, pero también es la explicación a la que se recurre para señalar deficiencias (como su falta de competencia lingüística o desconocimiento de las costumbres japonesas), porque no son japoneses. El discurso de la coexistencia multicultural, entonces, da cuenta de la diferencia, mientras ofrece medidas para facilitar la convivencia en las localidades

argumento del "hermano mayor" cuidando a los menores, Corea y Taiwán- (Oguma, 2002; McCormack, 2001). Oguma prueba que la reinvención de una nación pacifista y homogénea se da en la posguerra, no desde Meiji como otros autores sostenían. En el periodo previo a la guerra, en el que abundaban las explicaciones sobre la nación como multirracial, predominaban las teorías a favor de la diversidad étnica. Según el autor, con la derrota y la pérdida del imperio, Japón también pierde "diversidad étnica", los extranjeros son obligados a salir de Japón y los colonos japoneses diseminados por China y el sudeste asiático regresan a la tierra ancestral.

El caso de los nikkeijin es paradójico, pues a través de la modificación de 1990 a la Ley de Control a la Inmigración, la identidad nikkei ha sido empleada por el Estado para justificar convocarlos como similares étnicos, pero también es la explicación a la que se recurre para señalar deficiencias (como su falta de competencia lingüística o desconocimiento de las costumbres japonesas), porque no son japoneses. El discurso de la coexistencia multicultural, entonces, da cuenta de la diferencia, mientras ofrece medidas para facilitar la convivencia en las localidades. En la siguiente sección se aborda la cuestión de los debates por los derechos de las minorías en Japón. 


\section{Ciudadanía y extranjeros en Japón}

La presencia de migrantes extranjeros suscita debates por la delimitación de la ciudadanía (Méndez Vázquez, 2008). Ciudadanía es un término complejo que comprende la relación entre el individuo, el Estado y la sociedad, es un estatus concedido a los miembros plenos de la comunidad, cuya pertenencia implica tanto derechos civiles, políticos y sociales, como obligaciones (Marshall, 1992:18). Además del Estado y la comunidad local, el individuo pertenece a otros colectivos. Para dar cuenta de la pertenencia en los diferentes niveles, seguimos a Nira Yuval-Davis, quien concibe a la ciudadanía como "a multi-tier construct, which applies to people's membership in a variety of collectivities (local, ethnic, national and transnational)" (Yuval-Davis, 1997: 5). Así, podemos observar la relación entre el colectivo y el Estado, y la manera en que esta relación delimita la ciudadanía de las personas. ${ }^{11}$

Además de la pertenencia a una comunidad local y al Estado, hay otras divisiones sociales que atraviesan la ciudadanía, cuya incorporación a la misma fue promovida a mediados del siglo Xx por distintos movimientos sociales. ${ }^{12}$ Aun así, muchas de las elaboraciones posteriores continuaron modeladas sobre las sociedades occidentales. ${ }^{13}$ Si estos desarrollos se han producido en conexión con procesos acontecidos en Norteamérica, Europa o Australia, ¿qué ocurrió en Japón?

La literatura relevante sobre los extranjeros trataba hasta los años 1980 de los residentes de origen coreano (y de origen chino, descendientes de los

11. La ciudadanía, en la formulación de Marshall (1992: 8), posee aspectos civiles, políticos y sociales que se corresponden en determinados derechos civiles (propiedad privada, libertad de contratar), políticos (elegir y ser elegido) y sociales (vivienda, salud, educación, seguridad social). Mientras que la ciudadanía civil y política se vincula a derechos individuales, la social conlleva la participación en la comunidad, compartiendo un nivel básico de bienestar para todos, la herencia social que administra el Estado.

12. Mientras las democracias ensayaban formas distintas de incorporar a diferentes individuos en la nación, los estudiosos desarrollaron una ciudadanía sensible a la desigualdad derivada del género (Williams, 1998; Yuval-Davis, 1997), etnicidad (Kymlicka, 1989, 1995; Young, 1986, 1989), clase, estadio del ciclo vital y capacidades, entre otras divisiones, separándose de la tradición liberal de considerar a los ciudadanos como individuos con el mismo estatus y los mismos derechos y obligaciones.

13. Bottomore (1992) avanzó sobre la distinción de Brubaker (1989) entre derechos formales y sustantivos, mostrando cómo el movimiento transnacional de los trabajadores había contribuido al cuestionamiento de la pertenencia a un Estado-nación como la única fuente de ciudadanía. 
ex súbditos del imperio $)^{14} \mathrm{y}$ de los trabajadores migrantes, en términos de "el problema de los trabajadores extranjeros". Si se abordaba el género, se hacía desde la perspectiva del tráfico de mujeres, de las llamadas "novias asiáticas" y los matrimonios mixtos o kokusai kekkon. ${ }^{15}$

El concepto de ciudadanía que maneja el Estado japonés se asimila en principio a la relación unitaria (no se puede separar de la nacionalidad), homogénea (una sola cultura uniforme) y no mediada (se es o no se es ciudadano) descrita por Parekh (2000: 183). Un ejemplo de esto es la propuesta del ex director de la Oficina de Inmigración de Tokio Hidenori Sakanaka, quien aboga por un proyecto de permitir la entrada de 10 millones de extranjeros quienes serían naturalizados, como la solución a la crisis demográfica de Japón, con pocos nacimientos y una población envejecida (Sakanaka y Asakawa, 2007).

Enseguida se comentan autores que discuten el régimen japonés de ciudadanía y la situación de las mujeres migrantes.

Kibe (2006) considera que los originarios ainu moshir y los extranjeros coreanos zainichi son minorías etnoculturales, y propone que existen instituciones y prácticas de la ciudadanía de las cuales se pueden extraer principios de ciudadanía diferenciada para Japón. ${ }^{16}$ Esto ocurre a pesar del esfuerzo del Estado para preservar la homogeneidad cultural, la que el autor no acepta en términos de evidencia empírica y normativa. Él revela la brecha existente entre la práctica de la ciudadanía diferenciada y los intentos de los conservadores japoneses de reforzar la noción étnica de una identidad nacional. Kibe presenta la evidencia de los avances de la minoría ainu respecto a sus demandas de derechos culturales y sociales, y del poco éxito en el campo de los políticos (Kibe, op. cit.: 416). Asimismo, problematiza la ciudadanía y la relaciona con la desigualdad, señalando para el caso ainu que el reconocimiento de la diferencia cultural y la consecución de determinados derechos de ciudadanía, no solucionan per se la desigualdad social histórica, por lo que sostiene que la política del reconocimiento debería combinarse con políticas redistributivas (Kibe, 2006: 420 y 421).

14. Véase Kim (2006) para una explicación de las políticas para los residentes extranjeros, especialmente los coreanos en Japón, y a Chung (2010) para los logros del activismo de los residentes coreanos.

15. Con excepciones, como mi tesis de maestría sobre el tráfico de latinas para trabajo sexual, que analizó el fenómeno desde una perspectiva de género y derechos humanos (Méndez, 1998).

16. Los antecedentes de este término pueden encontrarse en la "ciudadanía diferenciada" de Young (1989), entendida como derechos culturales de un grupo específico. 
Para el caso coreano, propone que los reclamos de derechos culturales se han centrado en aspectos educativos, sus movimientos por derechos sociales se han beneficiado con la ratificación de las Convenciones Internacionales de Derechos Humanos en $1979{ }^{17}$ y la Convención sobre el Estatuto de los Refugiados en 1981. En cuanto a sus derechos políticos, los reclamos de los residentes coreanos han sido y son objeto de un debate social intenso. En resumen, el tipo de ciudadanía representada por los procesos en los que han participado los ainu es similar a la de los nacionales con derechos culturales especiales, mientras que los coreanos ejemplifican el estatus y la práctica similar a la del residente permanente o denizen (Hammar, 1990). Esto es, Kibe propone la posibilidad de una ciudadanía graduada y diferenciada, basada sobre evidencia empírica, la cual abre vías para la membresía y puede estar desconectada de la nacionalidad, apuntando a la pluralidad de culturas y afirmando positivamente los estatus que median entre las categorías de nacional y no-nacional (Kibe, 2006: 421).

Ito (2005) ha resaltado el vínculo entre género y ciudadanía, usando la categoría "familia" como un lugar privilegiado para el estudio de la estructura de las normas institucionales y los arreglos que regulan las relaciones entre ciudadanos y no-ciudadanos, lo que ella denomina el "régimen de ciudadanía". Su análisis expone la asimetría entre hombres y mujeres migrantes en el régimen de ciudadanía nipón, mostrando el proceso diferenciador que comienza en la forma de recepción en la sociedad japonesa de tanto hombres como mujeres migrantes. Esto es, ella distingue los estatus de visa disponibles para distribuirlos de manera diferente en el continuo de la ciudadanía. Explica que tanto hombres como mujeres pueden entrar a Japón con visas temporales (no calificados) y quedarse más allá del tiempo permitido, pero las mujeres también pueden ingresar por visas de artista o por vía del matrimonio. Al comparar la posición social y el lugar de las filipinas y brasileñas en la fuerza de trabajo, Ito encontró que las brasileñas estaban concentradas en los procesos de producción, mientras que las filipinas se hallaban mayormente en el sector servicios (con visas de artistas o de esposas de japonés) y también

17. Las convenciones mencionadas son el Pacto Internacional de Derechos Civiles y Políticos, y el Pacto Internacional de Derechos Económicos, Sociales y Culturales. Disponibles en:

http://www2.ohchr.org/spanish/bodies/hrc/index.htm http://www2.ohchr.org/spanish/bodies/cescr/index.htm http://www2.ohchr.org/spanish/law/refugiados.htm (Consultados el 25 diciembre de 2012). 
en la producción. Asimismo mostró que en cada caso "familia" podía ser alternativamente un aparato de subordinación racial y de género o un sitio de negociación, un recurso para reforzar la posición de ellas en el régimen de ciudadanía (Ito, 2005: 53 y ss.).

Tsuda (2006) sostiene que los países de inmigración reciente - y coloca aquí a Japón - se justifican en la no concesión de derechos a los inmigrantes, ya sea sobre la base de no ser países que tradicionalmente atraen inmigrantes o porque consideran que no hay urgencia para su integración sociocultural o política, presuponiendo su estatus temporario de residencia (Tsuda, 2006: 4 y 5). Y agrega que es más frecuente que esos países se centren más en controlar sus fronteras, que en conceder derechos de ciudadanía y atender las necesidades sociales de los extranjeros. ${ }^{18}$ Esto también se vincula con el hecho de que la Constitución japonesa garantiza los derechos sociales y civiles a los ciudadanos japoneses,$^{19}$ mientras que los estatutos sobre los gobiernos locales obligan a las autoridades locales a proveer la seguridad, salud y bienestar de todos los residentes locales (jûmin), incluyendo a los no-japoneses (Komai, 1997).

Tsuda ha sido criticado por no tener en cuenta lo suficiente la historia (el debate sobre la inmigración acompañó la empresa colonial nipona desde la Restauración Meiji) ni el rol de la minoría coreana en el desarrollo de la ciudadanía de los extranjeros en Japón (Chung, 2010). ${ }^{20}$ Tampoco incluyó el papel de los movimientos sociales liderados por otras minorías, como pueden ser los originarios ainu o los descendientes de los burakumin.

Un estudio publicado en 2001 por un grupo de expertos japoneses del Instituto Nacional para el Avance de la Investigación (NIRA), ya había anticipado la cuestión de la ciudadanía de los residentes extranjeros en Japón. Kajita (2001: 22) explica el rol del pasado colonial y las relaciones diplomáticas

18. Bottomore ha propuesto un corpus de derechos humanos para cada individuo, asegurando que los no-miembros del Estado-nación tengan la posibilidad de acceder a los derechos de ciudadanía en la comunidad donde viven o trabajan, independientemente de sus orígenes nacionales y su ciudadanía formal (Bottomore, 1992: 91 y 92). Si además coincidimos en que el Estado-nación ha sido la principal pero no la única institución que organiza la distribución de derechos y obligaciones de ciudadanía a los individuos, como lo han demostrado Hollifield (2000) y Joppke (1999), poseemos entonces las bases para comprensión de la ciudadanía local.

19. Véase Gurowitz (1999: 430) para la explicación de la interpretación de kokumin o pueblo (nación) en la Carta Magna japonesa como "gente del país", es decir, los nacionales japoneses.

20. El discurso oficial sobre la inmigración separa a los migrantes de la primera mitad del siglo XX u oldcomers de los más recientes o newcomers, como si la inmigración fuera de hecho un nuevo fenómeno. Aunque Chung (2010) no hace referencia directa a Tsuda (2006), ella incluye críticas a trabajos previos del autor. 
entre Japón y Corea, así como la importancia de la ciudadanía local (rôkaru shitizunshippu) como factor en el debate por la concesión de los derechos políticos a los extranjeros. Según el autor, "Mientras que a nivel del Estado las medidas son morosas, a nivel de las autonomías locales continúa la ampliación relativa de los derechos de los extranjeros y la teoría de la ciudadanía local (rôkaru shitizunshippu) en Japón se está volviendo un factor importante" (Kajita, 2001: 23). Concluye que ha llegado el momento en el que —además de las autonomías locales- el Estado asuma la solución integral del problema de los extranjeros. ${ }^{21}$

Para sintetizar, en esta sección se ha definido ciudadanía, cómo los modelos de ciudadanía se han desarrollado y tomado en cuenta el género y los no-nacionales, así como mencionamos literatura relevante en Japón. En la siguiente se discute el tipo de visa que se les concede a las mujeres y hombres nikkeijin, para comprender su situación dentro del régimen japonés de ciudadanía.

\section{Estatus de residencia de los nikkeijin y ciudadanía}

Desde la enmienda a la ley migratoria de 1990, la visa de residente por tiempo prolongado o visa de teijûsh $a,{ }^{22}$ bajo la cual los trabajadores latinoamericanos de origen japonés hasta la tercera generación han llegado a trabajar a Japón, les concede el derecho de emplearse en cualquier ocupación legal, lo que les da una posición de privilegio frente a otros residentes extranjeros. No obstante, los contenidos sustantivos de su estatus migratorio no están definidos. Siguiendo a Milly (2006: 127), presentamos el marco legal de los extranjeros hasta 2008, básicamente así:

There is no comprehensive piece of legislation in Japan that specifies all of the rights and policy entitlements of foreign residents, nor does a single coordinating

21. En el mismo volumen, Higuchi (2001: 40) se refiere a la ciudadanía local (emplea el término chihô shiminken), explicando que los coreanos residentes en Japón fueron en el pasado sujetos del Estado japonés y que luego de la derrota fueron privados de la nacionalidad, habiendo luchado por ampliar su ciudadanía en sus comunidades.

22. Atsushi Kondo (2001: 14) emplea el término "residentes cuasi-permanentes" indistintamente con el de long term o largo plazo, sobre la base de que aun cuando el período de residencia tiene que ser renovado periódicamente por los poseedores de la visa de largo plazo (teijûsha), ellos pueden trabajar sin restricciones, de la misma manera que los "residentes permanentes". Los plazos de renovación han sido para el periodo que nos ocupa de seis meses, un año o tres años. 
agency exists for policies affecting foreign residents. Instead, policies designed for Japanese citizens have produced policy-by-policy revisions, reinterpretations, or clarifications as needed when their applicability to foreign residents has been questioned. This means that advocates need to target a plethora of agencies and offices to work for policy change, and also that no institutional organ has an agency mission to oversee the inclusion of immigrants in domestic society and policies. [Las cursivas son nuestras.]

Este texto se publicó en 2006, y como se mencionó en la introducción, a posteriori el Gobierno japonés tomó su primera iniciativa para atender la situación de los extranjeros, particularmente el desempleo de los nikkeijin. El 9 de enero de 2009 se abrió no una agencia independiente, sino un buró dependiente de la Oficina del Gabinete del Primer Ministro con la misión explícita de elaborar medidas para los residentes extranjeros. Con la cooperación de los ministerios involucrados y para promover la implementación del apoyo a los gobiernos locales, produjeron unas directivas publicadas el 30 de enero de 2009, en las áreas de educación, trabajo, vivienda, apoyo al retorno y oferta de información. ${ }^{23}$ El buró coordina la elaboración interministerial de directrices, las que son presentadas a las autonomías (municipios y prefecturas) locales para su ejecución. En cuanto a la presión para cambiar las políticas, hay que acudir a los distintos organismos de acuerdo con la jurisdicción. Tampoco hay un organismo para supervisar la inclusión de los inmigrantes en la sociedad y en las políticas internas. Por todo lo anterior, si coincidimos en llamar "política de integración" a la política de derechos de ciudadanía de un Estado, podemos afirmar que en Japón no hay una política de integración social, sino una política de incorporación de extranjeros. Es una incorporación diferenciada que en principio extiende pero no garantiza ciertos derechos a ciertos grupos de extranjeros, con desarrollos particulares para los poseedores de la visa de residente permanente especial y los nikkeijin.

Veamos en la gráfica el comienzo del efecto de la crisis sobre la comunidad estudiada. El estallido de la crisis financiera contribuyó a la disminución del total de residentes peruanos registrados, pero no afectó la tendencia al establecimiento, que aún continúa (figura 1). La gráfica muestra la evolución hacia la residencia permanente que continuó incrementándose en 2008 y 2009,

23. Sobre el particular, véase la nota 2 . 
mientras disminuían el total y el número de peruanos con visa de residente por tiempo prolongado o largo plazo:

\section{Figura 1}

Peruanos con visa de largo plazo y de residente permanente

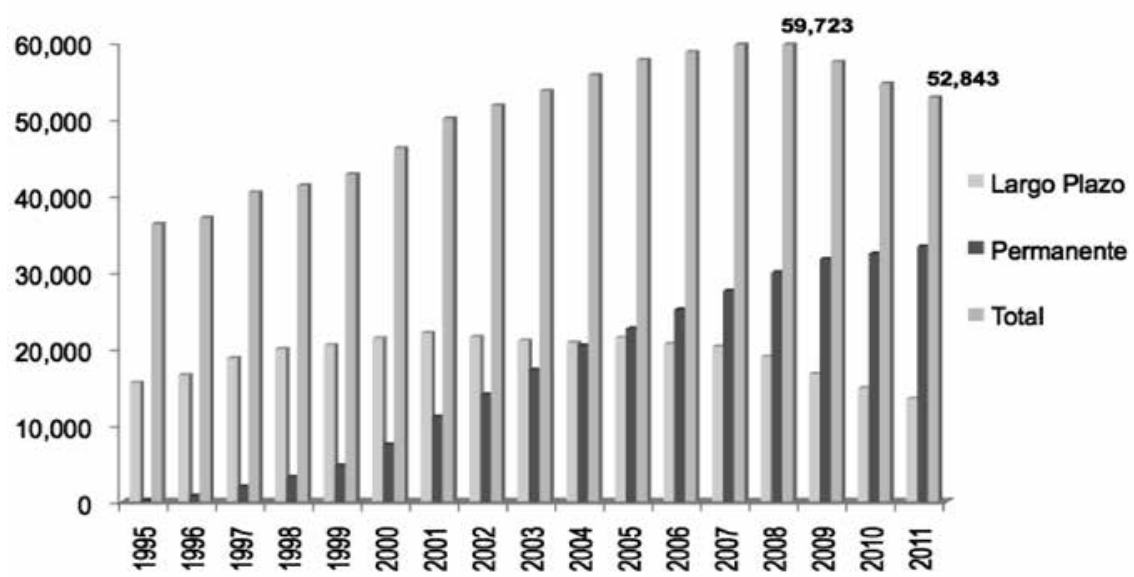

Fuente: Ministerio de Justicia de Japón, 20/02/2013; Censo Nacional 2005; Ministerio de Asuntos Internos y Comunicaciones.

Ha sido en el devenir de la residencialización que los trabajadores extranjeros emprendieron la búsqueda de ciudadanía a través de acciones específicas. En la siguiente sección vamos a presentar dos organizaciones de base, centrándonos en sus acciones hacia la expansión de sus derechos. ${ }^{24}$ Para esto hemos identificado instituciones cuyos agentes han producido cambios en sus comunidades. ${ }^{25}$ Agentes son las personas capaces de transformar sus condiciones de existencia, es decir, ellos realizan acciones con una "capacidad transformativa" (Giddens, 1981: 28). Pueden involucrarse en la acción en una comunidad ya sea permitiendo que se ejercite un poder a través de ellos (ellos median o permiten que ocurra una acción) o alcanzando un objetivo colectivo (lideran o provocan efectos).

24. La importancia de los grupos de base y las actividades comunitarias de las mujeres han sido tratadas por Yamanaka (2006), Kajita (1994, 2006); Komai (2001, 2006); y Yamawaki (2005), entre otros.

25. Para comprender cómo se han organizado estos grupos Cf. (2000), Linger (2001), Tsuda (2003), Roth (2002), Sakai (2006) y Córdova Quero (2008). 
Asimismo, como el Gobierno japonés reconoce como interlocutor a la Conferencia de Municipalidades con Concentración de Residentes Extranjeros, hemos comparado una organización en Kanto y otra en Tokai, una ubicada en un municipio que no pertenece a la Conferencia de Municipalidades con Concentración de Residentes Extranjeros, y una que no pertenece a esa organización suprarregional, pero que sí aprovecha esos recursos, ya que colinda con el municipio que sí lo hace.

En la figura 2 se muestran las ciudades miembro en 2007, el total y la proporción de extranjeros residentes, con las tres nacionalidades más frecuentes. ${ }^{26}$

\section{Figura 2}

Extranjeros y principales nacionalidades en la CMCRE

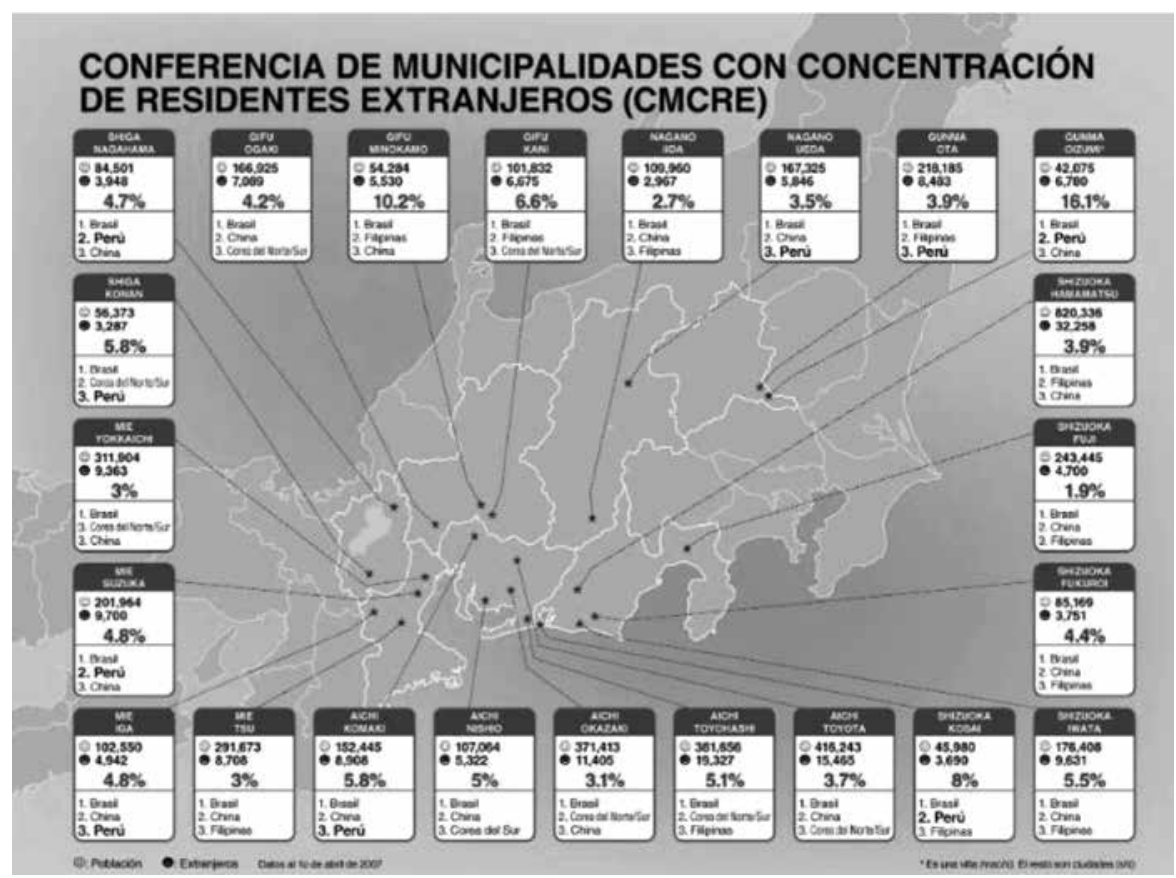

Fuente: CMCFP.

26. La organización se estableció originalmente el 7 de mayo de 2001, convocada por la ciudad de Hamamatsu, en la prefectura de Shizuoka. Su sitio es: http://www.shujutoshi.jp/ (Consultado el 5 de octubre de 2012). 
En esta sección se estableció que el marco de la coexistencia multicultural no garantiza per se la concesión de derechos a los extranjeros. A continuación se presentan dos asociaciones de peruanos, nikkeijin peruanos y japoneses, con foco en su acción colectiva.

\section{AJAPE O Nippon Peru Kyôsei Kyôkai}

La asociación fue creada oficialmente el 15 de agosto de 1999 en la ciudad de Machida, prefectura de Tokio, con el nombre de "Asociación PeruanoJaponesa" (APEJA) por un grupo de trabajadores y estudiantes peruanos, y nikkeijin de esa nacionalidad.

El grupo conserva su sede en Machida, una ciudad próspera en el extremo oeste de la prefectura de Tokio. Los miembros de AJAPE viven en su mayoría a lo largo del corredor de ciudades industriales que se alinean desde Sagamihara al norte, hasta Fujisawa al sur, en la prefectura de Kanagawa (figura 3).

\section{Figura 3}

Localización de AJAPE y área de influencia

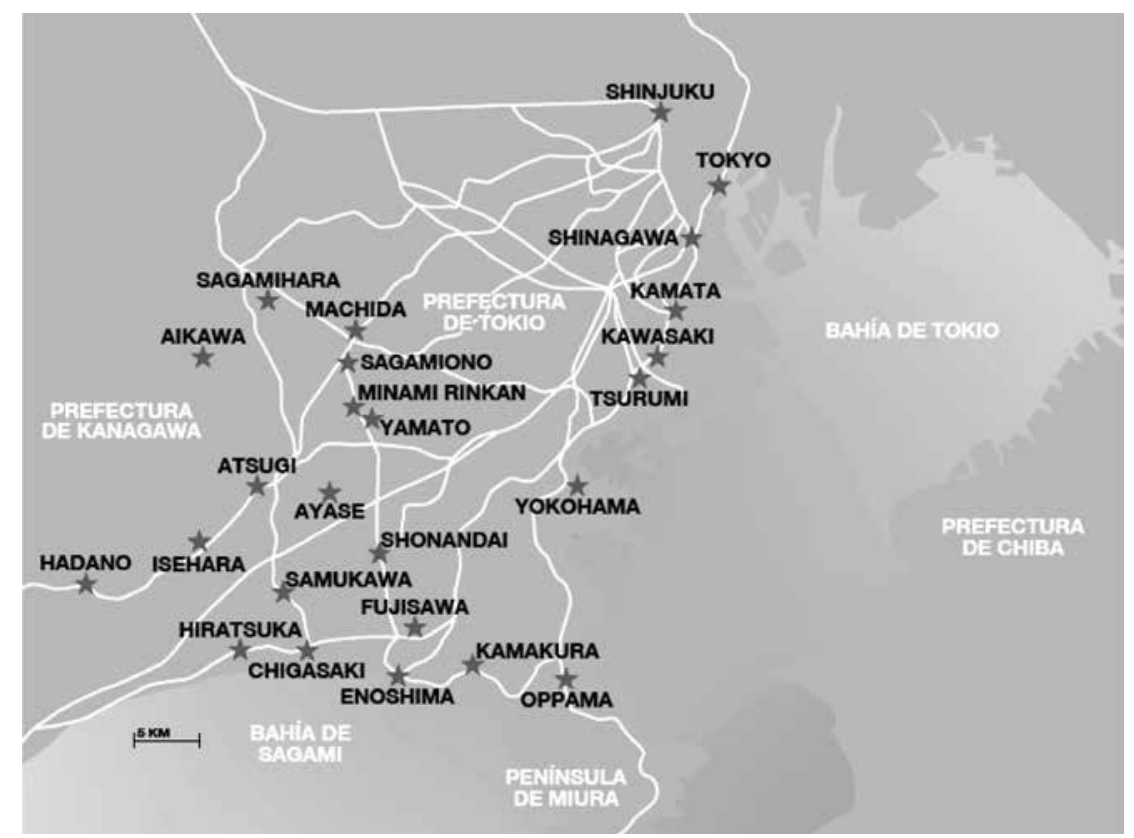

Fuente: autora. 
Antes de constituirse en una organización formal, eran en sus orígenes un núcleo de mujeres preocupadas por las dificultades que encontraban sus niños para adaptarse a las escuelas japonesas. Las mujeres buscaron apoyo y se reunían los fines de semana para acompañar a sus hijos en el aprendizaje del japonés, ya que muchos de los padres carecían de la habilidad lingüística y del conocimiento del sistema educativo. Pronto se incorporaron miembros japoneses, quienes se encargaron de asesorarlos en esos aspectos, entre ellos su vicepresidenta Etsuko Takahashi. ${ }^{27}$

La dinámica de la composición de sus integrantes provocó una fractura y algunos miembros de APEJA y sus líderes se separaron en busca de diferentes objetivos. Algunos de ellos no tenían su documentación en regla, por lo que dejaron el grupo, mientras que otros fueron deportados. Otra facción eligió participar en la política peruana y en la mejora de sus comunidades de origen, por lo que creó una nueva asociación: AIPEJA. Por su parte, el grupo de baile folclórico se separó para formar la compañía: "Así Es Mi Tierra" (AEMT).$^{28}$

En su quinto año (2004), APEJA cambió de nombre a AJAPE (Asociación Japonés-Peruana). ${ }^{29}$ Con este cambio sus miembros quisieron reflejar su deseo de integrarse e integrar a otros latinoamericanos a la sociedad japonesa, lo que ellos llaman "mejorar la calidad de vida" a través de los servicios educativos. ${ }^{30}$

27. Su presidente, el ingeniero peruano Alberto Palacios, no formó parte del núcleo original, pero ha estado a la cabeza de la organización desde el año 2000. Entrevista con S. S., miembro de AJAPE, septiembre de 2007.

28. Hasta que se separó de AJAPE, AEMT los representó exhibiendo folclor de Perú, Okinawa, Argentina, Bolivia, Chile y de Colombia. El original está en español, con fecha 3 de agosto de 2003.

http://ar.geocities.com/argentinayjapon/eventos/2003/08agosto-apeja.htm (Consultado el 25 de febrero de 2008).

29. 'Porque estamos en Japón y debería escribirse de esa manera. Lo nombras 'peruana' en primer lugar sólo si lo hubieras creado en el Perú. En algún momento del tiempo, las orientaciones de los miembros de AJAPE cambiaron de sus propósitos originales. Los miembros que no tenían una visa apropiada para permanecer en Japón se separaron. También cambió en ese momento el nombre de la asociación más antigua de los japoneses en Lima; la Sociedad Central Japonesa había cambiado su nombre en 1998 por el de "Asociación Peruano Japonesa" (APJ), el mismo nombre que nosotros habíamos elegido un año antes. Como ve, había muchos motivos para un cambio.” Entrevista con un miembro nikkei fundador de AJAPE, septiembre de 2007.

30. Sus propósitos expresados en sus estatutos son: 1. Mejorar la calidad de vida y promover la integración de peruanos y latinos a la sociedad japonesa; 2. Realizar eventos especiales con la finalidad de difundir las celebraciones y fiestas existentes en las diversas regiones del Perú y Japón; 3. Promover el conocimiento y el respeto de las leyes japonesas, realizando conferencias, seminarios, y todo tipo de actividades educativas para que los residentes peruanos y latinos en general conozcan sus derechos y obligaciones en Japón. 4. Promover el deporte en general, realizando eventos que sirvan para la integración y amistad entre Japón y Perú; 5. Promover la 
AJAPE funciona en un local que pertenece al municipio de Machida. Se definen como organización no gubernamental independiente de grupo religioso o político alguno. En 2008 contaban con tres empleados peruanos y cuatro japoneses apoyados por 12 voluntarios (cuatro latinos y ocho japoneses). Sus fuentes de financiación eran una anualidad de 2,000 yenes y fondos de la fundación The Nippon Foundation.

Tienen un servicio de consultas telefónicas sobre educación que atiende solicitudes de todo Japón. La profesora Takahashi proporciona información a los estudiantes y a sus familias. Ella publica sobre las actividades de APEJA y establece contactos con otros educadores, investigadores y voluntarios de otras organizaciones (Takahashi, 2007a, b).

AJAPE funciona los sábados y domingos y realizó en 2008 tres actos anuales, un taller de cultura, un festival internacional de danzas folclóricas y un foro sobre educación (figura 4$){ }^{31}$

\section{Figura 4}

Panel en el Forum AJAPE 2007

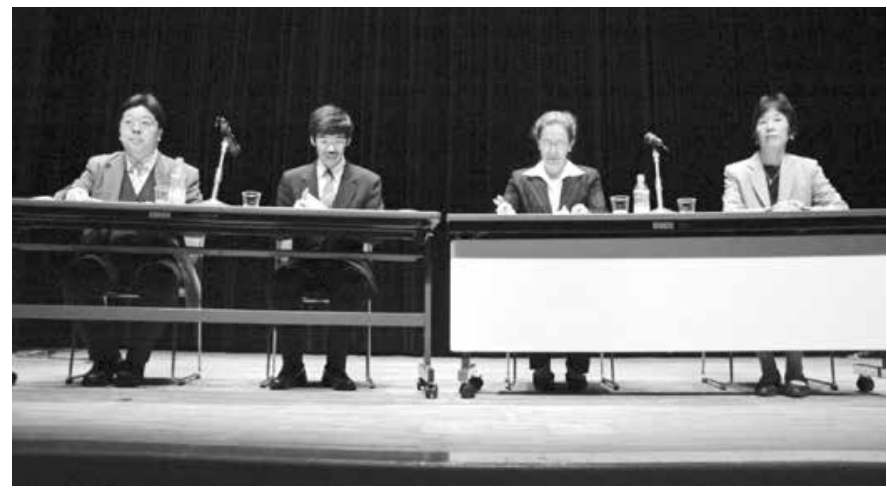

Fuente: autora.

enseñanza del idioma castellano entre los jóvenes peruanos y estudiantes japoneses; 6 . Promover la enseñanza del idioma japonés como medio ineludible de progreso en este país, 7. Promover actividades recreativas, sociales y comunales de diversa índole, acorde con los principios éticos y morales que todos tenemos. Original en español, fechado el 16 de septiembre de 2007.

http://ajape.org/index.php?option=com_content\&task=view\&id=2\&Itemid=3

(Consultado el 25 de febrero de 2008).

31. Apoyada nominalmente por la Embajada peruana y su Consulado general, son invitados a participar como representantes del folclor peruano. Desde 2005 hasta 2008 celebraron tres foros para discutir los tópicos de interés para la comunidad a la que sirven. 
Se pueden resumir los objetivos y actividades del grupo en cuatro áreas:

1. Mantener la lengua materna de los niños.

2. Mantener los lazos con sus países de origen.

3. Mantener los lazos con su comunidad local.

4. Reforzar el capital social de los niños y los adultos. ${ }^{32}$

Los niños y jóvenes apoyados por AJAPE son los familiares de los trabajadores nikkeijin. Los miembros permanentes de la asociación son unas 30 familias, que incluyen unos 100 residentes extranjeros. Además de peruanos, entre los usuarios de los servicios hay japoneses, filipinos, brasileños, mexicanos y otras nacionalidades. Resumiendo, el grupo proporciona instrucción de japonés y español para los niños de los inmigrantes y brinda a estos últimos clases de conversación en japonés.

Una de las características del grupo es su continuidad. Desde 1999 AJAPE ha sobrevivido a diversas circunstancias transformándose junto con su comunidad, apoyando a sus jóvenes miembros en su avance dentro del sistema educativo japonés. Este esfuerzo ha cambiado las perspectivas que aguardaban a los hijos de los trabajadores migrantes, quienes de otra manera hubieran abandonado sus estudios y no hubieran proseguido hacia la preparatoria y la universidad. ${ }^{33}$

Su otro rasgo distintivo es su proyecto de liderazgo. AJAPE ayuda a los niños y jóvenes a avanzar dentro del sistema, pero también intenta incentivarlos y darles conocimientos para facilitar su incorporación al mundo del trabajo. El proyecto los pone en contacto con empresarios japoneses, quienes comparten sus experiencias con los jóvenes en seminarios grupales. El programa compromete a los recientes graduados y empleados, a participar en las actividades de AJAPE a manera de devolución. Esta actividad se logra utilizando las redes personales de la vicepresidenta y del presidente de la asociación.

32. En términos simples, capital social remite a los recursos disponibles para un individuo en tanto miembro de un cierto grupo social, mientras el capital humano refiere a los atributos y habilidades de un individuo (Portes y Rumbaut, 2001).

33. La revisión a una ordenanza ministerial de fecha septiembre de 2003 concerniente al examen de ingreso a la universidad, permitió que los graduados extranjeros de preparatoria pudieran postularse a los exámenes de admisión. Desde entonces la asociación ha mediado exitosamente para obtener el reconocimiento de los certificados de educación a distancia de Perú como prueba de capacidad, porque como ha dicho un miembro: "en la mayoría de los hogares no hay dinero extra para pagar un juku (academias particulares para preparar los exámenes) para entrar a la secundaria superior". Entrevista con M. F., ciudad de Yamato, prefectura de Kanagawa, diciembre de 2007. 
A continuación se presenta la asociación de creación más reciente: APEAA.

\section{APEAA O Aichi Perû Bunka Kôryû Kyôkai Apeaa}

La agrupación fue creada oficialmente en octubre de 2007 como la Asociación Peruana de Aichi "Apoyo" o APEAA, por un grupo de 10 familias de trabajadores migrantes de Perú, un total de 60 personas.

En 2008 funcionaba en las instalaciones del centro comunitario de Inuyama (Inuyama Fureai Sentâ), cercano al complejo habitacional Gakuden, hogar de unas 150 familias peruanas (figura 5).

Figura 5

APEAA y área de influencia

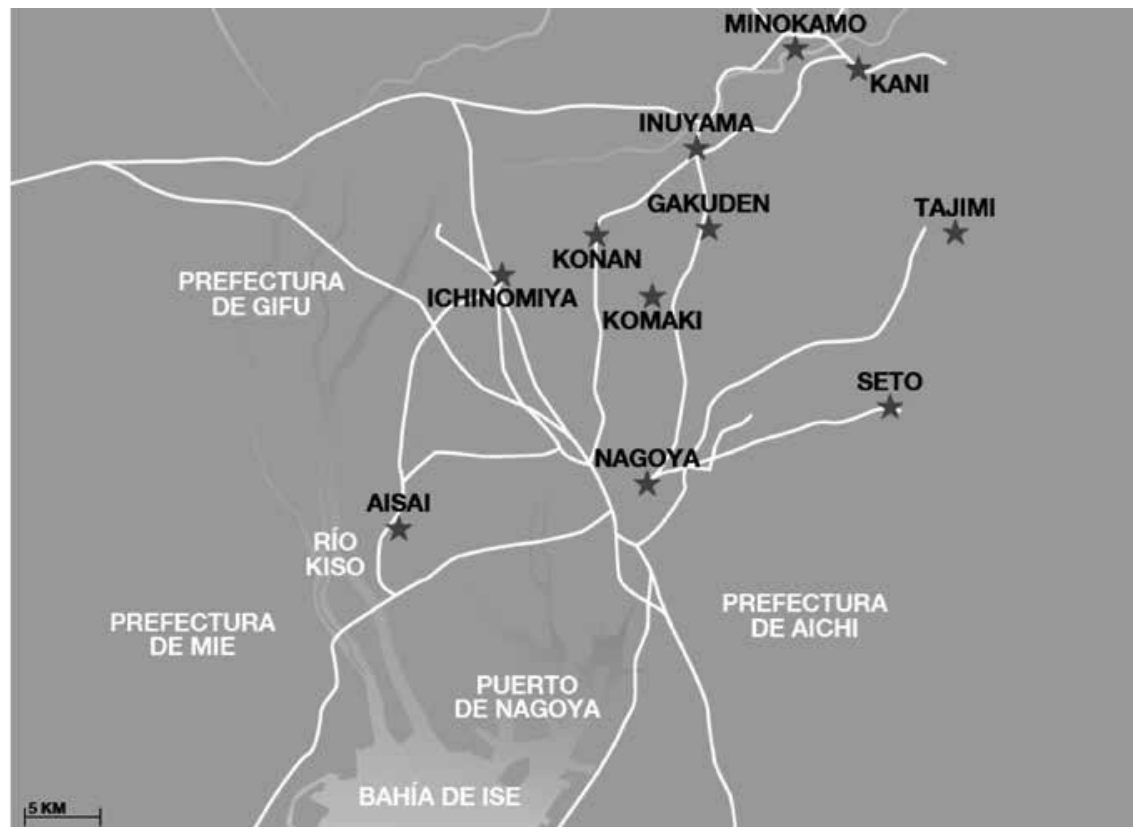

Fuente: autora.

Según uno de sus miembros fundadores, antes de tener una estructura definida comenzaron como un grupo de familias lideradas por madres que estaban preocupadas por el avance de sus hijos en la escuela y por encontrar formas de complementar el ingreso doméstico vendiendo platos peruanos a 
otros trabajadores. ${ }^{34}$ Entonces, Maritza Hirose, una trabajadora nikkeijin con buen dominio del japonés hablado, se organizó con otras madres para buscar voluntarios de oenegés locales que quisieran apoyar a sus hijos.

El terremoto que destruyó la ciudad peruana de Cañete el 15 de agosto de 2007, los impulsó a constituirse en una organización. Ese mismo día se propusieron comenzarla con el propósito extraordinario de reunir recursos para los afectados y con el objetivo fundamental de asistir a las familias de los asociados en las cuestiones educativas. De acuerdo con un miembro, fue

\begin{abstract}
[...] una tarea difícil, porque estábamos plenamente empleados y nadie tenía ni el tiempo ni la voluntad de reunirse para elaborar los estatutos, bajar hasta Nagoya a registrarse en el Consulado peruano y hacer reconocer nuestra asociación ante las autoridades japonesas. Tampoco teníamos un intérprete de español en las dependencias públicas de la ciudad de Inuyama en ese momento. ${ }^{35}$
\end{abstract}

Su presidente, Ricardo Ruiz, confirmó que él y la encargada de las actividades educativas y de prensa, Maritza Hirose, desarrollaban gran parte de las tareas junto con su vicepresidente, aunque contaban con el apoyo de otros miembros.

A lo largo de 2008 se consolidó la organización para llegar a otros peruanos y para acercarse a sus vecinos japoneses a través de festivales, puestos en ferias organizadas por la oenegé japonesa "Shake Hands", espectáculos de folclor peruano, música y gastronomía de otros países latinoamericanos. Con el dinero recolectado pudieron desarrollar sus propias actividades. ${ }^{36}$ "Para ganarnos un lugar, ciertamente intentamos borrar la mala impresión que otros miembros de la comunidad peruana habían dejado en los japoneses", aseguró la señora M. S. ${ }^{37}$

La crisis de créditos global obligó a renunciar a algunos miembros hacia finales de 2008, cuando perdieron sus empleos, y buscar soluciones individuales desesperadamente. La venta de platillos peruanos y las clases de cocina se volvieron una importante — cuando no la única — fuente de ingresos para las familias de los miembros que recibían o no su seguro de desempleo. ${ }^{38}$

34. Entrevista con M. S., ciudad de Inuyama, prefectura de Aichi, 2 de junio de 2008.

35. Entrevista con A. R., ciudad de Inuyama, prefectura de Aichi, 2 de junio de 2008.

36. El centro comunitario de Inuyama no les cobraba el espacio, sólo por reserva con anticipación, presentando el programa de actividades.

37. Entrevista con M. S. vía Internet, 15 de junio de 2009.

38. Entrevista con A. R., vía Internet, 16 de junio de 2009. 
Asimismo debieron cancelar algunas de las actividades programadas conjuntamente con la organización no gubernamental japonesa "Shake Hands", liderada por Satomi Matsumoto, una residente local muy activa, fomentando el contacto entre diferentes asociaciones para extranjeros. Igualmente, en febrero de 2009 y para ajustarse a la difícil situación económica cambiaron los estatutos de APEAA, estableciendo que su principal propósito es "promover la integración entre los peruanos, su integración a la sociedad japonesa y su integración a otras comunidades que viven en el territorio japonés".

Con el apoyo de la trabajadora Yumi Oshima, una voluntaria nikkeijin brasileña, intérprete de portugués y español y miembro del Comité Consultivo de la ciudad de Komaki (Komaki Kondankai), ${ }^{39}$ APEAA se registró en el municipio de esa ciudad contigua a Inuyama. ${ }^{40}$

Las actividades regulares de APEAA en 2008 incluían:

- Lengua japonesa para niños de escuela primaria y secundaria.

- Lengua japonesa para padres (con el apoyo de Shake Hands y del Centro de Inuyama para el apoyo local).

- El programa de radio "La hora latina" en la emisora de FM 842 (http:// www.842.fm) (figura 6).

Asimismo organizaron actividades extraordinarias:

- Festival de la Amistad Latina, marzo de 2008.

- Torneo de Futbol Sala, en mayo de 2008.

- Primer evento educativo: "Taller de inmigración e identidad", junio de 2008.

Y participaron en los actos:

- "Harvest Thanksgiving Festival" en Freude, la Asociación Internacional de Inuyama, 20 de agosto de 2008.

39. Ese cuerpo consultivo con los extranjeros celebró su primera reunión fundacional el 6 de julio de 2008, sesión a la que asistimos.

40. Esto ocurrió luego de nuestra investigación, el 28 de abril de 2009, en plena crisis financiera. Fue el primer paso de la organización para formalizarse frente a las autoridades locales, manifestar su interés en tomar parte en las actividades para extranjeros y recibir apoyo del municipio, como fue propuesto en las directrices del Gobierno central a comienzos de ese año. Entrevista con R. R., vía Internet, 16 de junio de 2009. 


\section{Figura 6}

El presidente de APEAA transmitiendo "La hora latina" (2008)

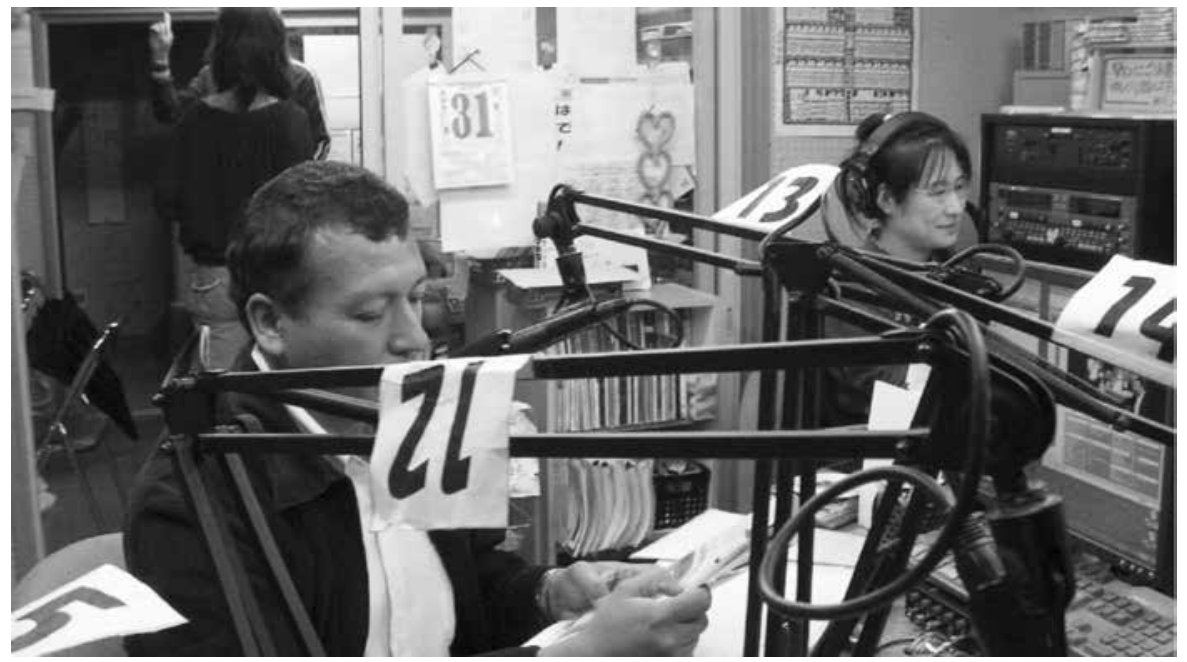

Fuente: autora.

Resumiendo, el grupo se moviliza en principio para proporcionar instrucción de japonés y apoyo escolar para los niños de los inmigrantes y les brinda a los padres clases de conversación en japonés. Para funcionar ellos precisan realizar actividades de recaudación de fondos, como su participación en festejos locales, su propio festival de la amistad o su torneo de futbol. ${ }^{41}$

Una de las características de este grupo es su flexibilidad. Esto debido a que es de creación reciente y que aún no logra estabilizarse. ${ }^{42}$ Su otro rasgo distintivo es que, si bien tenían contacto con las oenegés locales al momento del trabajo de campo, no tenían miembros japoneses ni habían considerado su inclusión necesaria o deseable a corto plazo. Las mujeres no ocupan los

41. En comunicación personal, luego de concluido nuestro trabajo de campo, también refirieron otras actividades para recabar fondos durante la crisis, a saber: su participación en una "Exhibition of Peruvian Crafts and Pisco Tasting” en Freude, la Asociación Internacional de Inuyama, el 7 de marzo de 2009 y clases de culinaria peruana para 25 estudiantes japoneses el 8 de marzo de 2009. Entrevista con R. R., vía Internet, 16 de junio de 2009.

42. Durante la crisis en 2009, su presidente me consultó sobre la posibilidad de organizar una colecta para las familias desempleadas de APEAA en ocasión de mi presentación en un congreso. Cabe mencionar que esa colecta no se realizó, con el consiguiente descontento de algunos miembros de la organización. 
máximos cargos en esta organización y se encargan de roles vinculados a la educación, difusión y culinaria.

Hasta aquí hemos presentado la actividad de la asociación APEAA de Inuyama, Aichi. En la sección final se contrastan las actividades en procura de ciudadanía a nivel local y el rol de las mujeres en las asociaciones.

\section{Sumario. Conclusiones}

Las actividades concebidas por AJAPE (Kanto) y APEAA (Tokai) han permitido la integración de algunos individuos al sistema educativo y al empleo en sectores distintos de la manufactura. En ambos grupos han ejercitado la ciudadanía a nivel local por medio de acciones colectivas que ambas asociaciones han coordinado, es decir, han sido agentes de cambio entre sus miembros. Aun con resultados favorables a nivel micro, es válido preguntarse por la efectividad de los esfuerzos de capacitación de la lengua y apoyo educativo para mejorar la calidad de vida de los extranjeros. Elevar la calidad de vida implica, entre otras cosas, un nivel de ingresos mayor. Takenoshita (2006: 70-74) muestra dentro de la teoría del capital humano que los resultados dependen de cómo los inmigrantes son tipificados, esto es, de cuál segmento dentro del mercado de trabajo se considera que ocupan. Compara entonces la adquisición de capital humano y su impacto en los niveles de ingreso de trabajadores manuales brasileños y profesionales chinos en Japón. Los teóricos del capital humano suponen que mientras los inmigrantes adquieran competencia lingüística, completen niveles educativos y logren certificaciones en su empleo, ellos podrán equipararse en los niveles salariales de la sociedad que los acoge. Takenoshita prueba que sería difícil para los trabajadores manuales brasileños elevar su nivel de ingresos aun si hablaran más japonés, completaran un nivel superior de educación y permanecieran más tiempo en Japón. Si no es su capital humano, ¿qué impide que mejore su salario? Takenoshita (2006: 71-73) sostiene que más que su adquisición de capital humano, tiene más impacto en determinar los ingresos de los trabajadores manuales brasileños la demanda laboral en la compañía y sector del mercado de trabajo que ocupan. Dicho de otra manera, los ingresos están limitados por el tipo de incorporación, el segmento del mercado de trabajo que ocupan los trabajadores brasileños y peruanos en su mayoría. Las posibilidades de mejora en la calidad de vida se abrirían con la transición del empleo indirecto a través de brokers al empleo directo por las compañías. En ese punto podrían 
incrementarse las oportunidades de capacitación ofrecidas por los empleadores y mejores niveles de salario.

La existencia de resultados exitosos en las comunidades estudiadas no cancela el hecho de que los alcances de la ciudadanía local son limitados (Andrew y Goldsmith, 1998; Tegtmeyer Pak, 2001; Tsuda, 2006; Méndez Vázquez, 2008). A pesar de ofrecer derechos sustantivos y obligaciones, la ciudadanía local está lejos de ser el objetivo último del grupo o de alguno de los colectivos de extranjeros en Japón. Al ser local, no es uniforme ${ }^{43}$ y tampoco está sustentada por recursos comparables a los del Estado nacional (Chung, 2010).

AJAPE es fundamentalmente un grupo de la prefectura de Kanagawa, si bien está ubicado en la prefectura de Tokio y usa recursos del municipio de Machida con el apoyo de su vicepresidenta y con la figura de su presidente. La mayoría de sus miembros viven en las ciudades fabriles de las áreas arriba descritas. Esta asociación tiene miembros japoneses y una relación estrecha con el municipio y la comunidad, a través de las redes y el capital social de sus directivos. Asimismo gestionan fondos de oenegés japonesas, por lo que tienen un reconocimiento más allá del ámbito de la autonomía local que los acoge.

APEAA es sin duda un grupo de la ciudad de Inuyama, pero que aprovecha los recursos de la ciudad de Komaki, la cual es de mayores dimensiones y con mayores recursos. Porque pertenece a la Conferencia de Municipalidades con Concentración de Residentes Extranjeros (CMCRE), tiene servicios de los que Inuyama carece (intérpretes de español y portugués en los hospitales y otros organismos públicos, información y folletos plurilingües, empleados en el municipio que hablan portugués o español, etcétera). De creación reciente al momento del trabajo de campo, no tenía miembros japoneses y no estaba registrada en el municipio en el que tiene sede. ${ }^{44}$ Tampoco recibía fondos de organización alguna, pero sí realizaba tareas conjuntamente con la oenegé japonesa mencionada. Para su financiamiento dependía exclusivamente de los fondos autogenerados.

43. Saruhashi (2007: 54) centra su estudio en los servicios lingüísticos del progresista municipio de Kawasaki; afirma que en muchos municipios la sola provisión de servicios en otras lenguas que no sea japonés es vista como una carga molesta (mendôna futan), y el propio servicio, como algo redundante (ekusutorana).

44. Arriba mencionamos que esto tuvo lugar en 2009, cuando el desempleo rampante afectó la comunidad. Se registraron para beneficiarse del apoyo en la emergencia del gobierno local (como los cursos de capacitación laboral para los desempleados, por ejemplo). 
Una de las características halladas es que la ciudadanía local en el contexto de la coexistencia multicultural japonesa tiende a traducirse en la provisión de servicios a los extranjeros locales, más que a garantizar sus derechos sustantivos - ya sean básicos o sociopolíticos-. Además es evidente que las oenegés y las actividades de los extranjeros en la comunidad ayudan a resolver los problemas de algunos, pero no logran atender las necesidades educativas y lingüísticas del colectivo. Como ha señalado Yorimitsu (2006: 207), Japón no es una sociedad monoétnica y como sociedad multicultural requiere una organización especializada; el apoyo de los extranjeros no puede ser dejado solamente a voluntarios; en esta línea, la política de coexistencia multicultural debería ser considerada de la mayor importancia para el Estado nacional.

En segundo lugar, es notable que los esfuerzos a nivel local son dispares e insuficientes tanto del lado proveedor del servicio educativo (coordinado por el comité educativo local en cada municipio), como del lado de los inmigrantes. Esto se demuestra en el hecho de que en ambas regiones es necesario el apoyo escolar y lingüístico para los niños y -como hasta ese momento sólo había disponibles algunas clases en las asociaciones internacionales locales- el apoyo de idioma para padres. Del lado de los inmigrantes, se ha visto la diferencia en los términos de apropiación de la ciudadanía en cuanto al derecho a la educación, de acuerdo con las limitaciones y capacidades (recursos) de cada asociación. En el caso de APEAA inclusive, las actividades en pro de la educación de los niños y la capacitación de los padres, en medio de la crisis se vieron supeditadas a la necesidad de recaudar fondos para las familias cuyas cabezas habían perdido el empleo, y así actuar en esa coyuntura más como una sociedad de socorros mutuos de facto, tradicional en la literatura sobre migrantes, que como un grupo orientado a la educación e integración.

En tercer lugar, la forma de manejar los recursos disponibles de ambas asociaciones confirma que no podemos contemplar a la comunidad como algo dado. Las colectividades y las comunidades son también construcciones ideológicas y materiales cuyos límites, estructuras y normas son el resultado de procesos y negociaciones (Eriksen, 2002). Que los nikkeijin hayan sido considerados por el Estado japonés un colectivo apto para gozar de un estatus migratorio especial con base en una similitud étnica, fue una construcción particular de ellos como trabajadores privilegiados, una comunidad distinta a incorporarse en las áreas donde eran requeridos.

Asimismo, la incorporación de los nikkeijin peruanos y demás miembros de estas asociaciones a sus comunidades locales a través de la educación (y 
de actividades sociales recreativas o artísticas), depende mucho de su propio esfuerzo, pero además, las "comunidades" no están demarcadas necesariamente por términos municipales u otra división administrativa. Su estrategia de manejo de los recursos disponibles lo muestra. En última instancia, el objetivo de los padres nikkeijin peruanos es que sus hijos avancen exitosamente en el sistema educativo japonés, y se puedan integrar al mercado laboral en circunstancias mejores que las propias, a la comunidad mayor de la sociedad japonesa. ${ }^{45}$

Hay otro sentido en el que los límites de la comunidad cambian, vinculado a la competencia por los puestos de trabajo. Se ha visto que en ambas asociaciones no sólo hay miembros nikkeijin peruanos: también las integran otras nacionalidades. Empero, en el caso de APEAA se da una situación frecuente en áreas industriales: la mayoría de nikkeijin en Komaki es de brasileños, con quienes los nikkeijin peruanos compiten en el mercado laboral, el cual se mantiene segmentado y cuyo acceso se produce a través de brokers que sólo operan con una nacionalidad exclusivamente y no con otra (Roth, 2002). Al lograr el apoyo de nikkeijin brasileños, más capacitados y con más conocimiento operativo de las instituciones locales, APEAA ha modificado los límites de una estrecha comunidad de peruanos en beneficio de sus miembros.

Los trabajadores perciben: "Porque los brasileños son más y saben más japonés que nosotros, pueden negociar mejor" ${ }^{46}$ Los autores coinciden en que no sólo hay una jerarquía de nacionalidades y razas para los japoneses (Shipper, 2002, 2008; Tsuda, 2009) ${ }^{47}$ sino también una jerarquía étnica operando dentro de la categoría nikkeijin (Takenaka, 2009; Tsuda, 2009), por la que los brasileños gozan de un estatus superior a los peruanos. Según Takenaka (op. cit., 266):

[...] Brazilians maintained, and were considered to maintain, more Japanese cultural and ethnic features than Peruvians on average and were subsequently ranked higher than Peruvians in the ethnic hierarchy in Japan. And this was reinforced by the higher status of Brazil in the global hierarchy of nations, compared to Peru.

45. Entrevista con M. O., ciudad de Komaki, prefectura de Aichi, 24 de agosto de 2008.

46. Entrevista con A. R., Inuyama, prefectura de Aichi, 2 de junio de 2008.

47. Tsuda (2009: 228 y 229) explica cómo los nikkeijin peruanos están considerados en un rango superior a los brasileños, mientras Shipper (2008: 57) sostiene que la política migratoria japonesa distribuye a los trabajadores extranjeros de acuerdo con razas y nacionalidades. 
Coincidimos con Takenaka (2009) ${ }^{48}$ en que la migración étnica no conlleva el surgimiento de la solidaridad étnica, frecuentemente lleva a diferenciación y a fracturas en el grupo. De aquí se considera un pequeño logro para APEAA el que haya conseguido el apoyo de brasileños con mayor conocimiento de las instituciones y redes locales, expandiendo su propia red de relaciones hacia la integración local.

En cuarto lugar y con referencia a las mujeres y su agencia para obtener ciudadanía local a través de las actividades comunitarias, como se desveló durante la estancia de campo, el hallazgo coincide con lo que Pierrette Hondagneu-Sotelo (1994) definió como la actividad para la consolidación del asentamiento (consolidate settlement). Esto significa que a través de la experiencia migratoria, el rol tradicional de las mujeres cambia al debilitarse los roles patriarcales tradicionales en las comunidades de origen. Las mujeres asumen roles públicos más dinámicos, activan y crean redes y aseguran formas de asistencia para sus familias, las cuales son cruciales en el proceso de residencialización.

A lo largo de las acciones de las mujeres migrantes para la incorporación y consecución de la ciudadanía de sus familias, los roles de género son negociados en lo que Yamanaka (2003: 169) describió como el proceso en el que madres que eran "amas de casa y brindadoras de cuidados", y padres que "se ganan el pan" negocian roles que se vuelven más y más complejos. En nuestra investigación surgió que de esa negociación hay mujeres que se han convertido en cabeza de familia, porque por ejemplo se han divorciado, una opción impensable en algunas de sus "conservadoras comunidades nikkeijin", mientras que otras han abierto espacios para desarrollar actividades más allá del cuidado del hogar. ${ }^{49}$

Por otra parte, las actividades implementadas por las trabajadoras peruanas en apoyo de la educación de sus hijos no son un caso aislado: este tipo de actividades también ha sido notada para el caso de los residentes nikkeijin brasileños por Yamanaka (2004). Nuestro caso prueba que las iniciativas de las mujeres vienen a corroborar una situación estructural, es decir, una defi-

48. Su pertinente análisis da cuenta de las diferencias de clase y raza dentro del mismo grupo de los nikkeijin peruanos en Japón (Takenaka 2009: 275), diferencias con las que coincidimos pero que están más allá del alcance de este artículo.

49. Una de las entrevistadas en Tokai se describió en ese momento como "menos ama de casa y más una cineasta", porque "yo negocié este espacio". Entrevista con M. O., ciudad de Komaki, prefectura de Aichi, 24 de agosto de 2008. 
ciencia del sistema japonés en relación con la integración de los extranjeros. Finalmente, no queremos dar aquí una visión extremadamente positiva de la experiencia migratoria de las mujeres: se trata de posibilidades que se abren y que no existían, según las entrevistadas, en las comunidades de origen. No sólo son las trabajadoras nikkeijin y otras extranjeras las agentes en estas actividades de base: empleadas japonesas de los municipios y miembros de las oenegés niponas se reparten la tarea de apoyar a los extranjeros, con lo que reafirman su rol de género de "encargadas de la educación" de los niños (Yamanaka, 2006)..$^{50}$

En quinto lugar, en nuestra comparación entre las dos instituciones de las dos regiones se ha mencionado que sí hay diferencias en los servicios y recursos disponibles en municipios de acuerdo con sus dimensiones, pero no se encontraron diferencias específicas o inherentes a la pertenencia a la Conferencia de Municipalidades con Concentración de Residentes Extranjeros, que pudieran distinguirse muy particularmente de los servicios disponibles, por ejemplo en Kawasaki o Machida, ciudades fuera de la Conferencia. Queda este punto para investigación ulterior.

Por otra parte, estas mujeres trabajadoras también ponen de manifiesto lo tenue de la multiculturalidad japonesa. Esto es, que si bien es cierto que sólo se considera el idioma japonés a la hora de brindar algún apoyo lingüístico, éste ni siquiera es uniforme en todo el país y, por lo tanto, no hay un contradiscurso "hacia una política asimilacionista": son los mismos extranjeros quienes se organizan para promover el avance de sus hijos dentro de la educación obligatoria en la lengua nacional, mientras que también procuran asistencia para mejorar el nivel de español de sus retoños.

En sexto lugar, el multiculturalismo planteado por el Estado, la coexistencia multicultural, puede parecer un desafío a la ideología de Japón como nación homogénea, pero no es muy diferente del discurso asimilacionista de la época del Imperio japonés (Tai, 2007: 21). El discurso oficial de la coexistencia multicultural es una forma propia de discurso nacionalista (Lee, 2003: 103) en la que se protege la homogeneidad y los límites del Estado. Contemporáneamente, los extranjeros están privados de algunos derechos clave (elegir y ser elegidos), pero se les reconocen otros (acceso a la vivienda pública, seguro de salud); antaño partícipes de una esfera de co-prosperidad panasiática, se

50. Yamanaka publicó en 2004 su investigación acerca de un grupo de brasileñas nikkeijin que en 1996 ya se había organizado para apoyar la educación de sus hijos en Hamamatsu, Shizuoka. 
los reconoce ahora como residentes integrantes de la comunidad local, con acceso a ciudadanía, que se ha ido forjando a través de movimientos sociales ya mencionados, anteriores a la llegada masiva de los nikkeijin en torno a la última década del siglo $\mathrm{xx}$.

La hegemonía de un discurso monocultural ha sido reemplazada por la apropiación por parte del Estado de un discurso que alude a la multiculturalidad como diversidad étnico-cultural, desprovista de todo vínculo o crítica de la desigualdad, ${ }^{51}$ pero ligado a la provisión discrecional de derechos civiles y sociales.

Finalmente, cabe afirmar que en el periodo estudiado el rechazo oficial a una política de inmigración y las políticas para extranjeros fundamentalmente de servicios, (mayormente para nikkeijin) agrupadas bajo el rótulo "coexistencia multicultural", no cancelan el hecho de que todavía están por formularse en Japón unas políticas extensas para la integración de los extranjeros, con la participación de japoneses y extranjeros.

\section{Referencias bibliográficas}

Ando, Mitsuyo, y Kimura, Fukunari (2012), "How did the Japanese Exports Respond to Two Crises in the International Production Networks? The Global Financial Crisis and the Great East Japan Earthquake”, Asian Economic Journal, 26(3), pp. 261-287.

Andrew, Caroline, y Goldsmith, Michael (1998), "From Local Government to Local Governance — and Beyond?”, International Political Science Review, 19(2), pp. 101-117.

Bottomore, Tom (1992), Citizenship and Social Class, Londres: Pluto Press, pp. 85-139.

Brubaker, William Rogers (1989), Immigration and the politics of Citizenship in Europe and North America, Nueva York: University Press of America.

Chung, Erin Aeran (2010), Immigration and Citizenship in Japan, Nueva York: Cambridge University Press.

51. Ryang (2008, op. cit.), como nosotros, remarca en el caso de los coreanos que para el Estado japonés es totalmente ignorada la cuestión de clase, ya que (como a otros extranjeros, inmigrantes, o refugiados) no se les da lugar en la estratificación de clase doméstica del Estado-nación. Asimismo agrega que factores como la pobreza — que podrían llevar a formaciones de clase- son constantemente etnicizados. 
Córdova Quero, Martín Hugo (2007), "The Role of Religion in the Process of Adaptation of Brazilians of Japanese Ancestry to Japanese Society: The Case of the Roman Catholic Church", en Mita, Chiyoko, Córdova Quero, Hugo, Litvin, Aaron, y Haino, Sumiko (eds.), Sociedade Japonesa e Migrantes brasileiros: Novos Caminhos na Formação de uma Rede de Pesquisadores, Tokio: Sophia University-Center for Luso-Brazilian Studies, pp. 93-104. (2008), "Encounter between Worlds: Faith and Gender among Japanese Brazilian Migrants in Japan", The Journal of Sophia Asian Studies, núm. 26, pp. 1-18.

Doak, Kevin M. (2007), A history of nationalism in modern Japan. Placing the people. Leiden, Boston: Brill.

Eriksen, Thomas Hylland (2002), Ethnicity and Nationalism, Londres: Pluto Press.

Giddens, Anthony (1981), A Contemporary Critique of Historical Materialism, vol. 1., "Power, Property and the State", Londres: Macmillan.

Glick Schiller, Nina (2005), "Transnational urbanism as a way of life: A research topic not a metaphor”, City \& Society, 17(1), pp. 49-64.

Gurowitz, Amy (1999), "Mobilizing International Norms: Domestic Actors, Immigrants, and the Japanese State", World Politics, 51(3), abril, pp. 413-445.

Hammar, Tomas (1990), Democracy and the Nation State: Aliens, Denizens and Citizens in a World of International Migration, Brookfield, vT: Averbury.

Hanazaki, Kôhei (2002), Kyôsei e no shokuhatsu: datsu shokuminchi, tabunka, rinri o megutte [Inspirando la coexistencia: En torno a la ética de la descolonización y la multiculturalidad]. Tokio: Misuzu Shobo.

Higuchi, Naoto (2001), "Gaikokujin sanseikenron no nihonteki kôzu: shiminkenron karano apurôchi”, en NIRA Citizenship Kenkyukai (ed.), Tabunkashakai no Sentaku: Shitizunshippu no Shiten kara, Tokio: Nihonhyoronsha.

Hollifield, James (2000), "The politics of international migration: How can we "bring the State back in", en Brettell, Caroline B., y Hollifield, James F. (eds.), Migration theory: Talking across disciplines, Nueva York/Londres: Routledge.

Hondagneu-Sotelo, Pierrette (1994), Gendered Transitions: Mexican Experiences of Immigration, Berkeley/Los Ángeles, California: University of California Press. 
Ito, Ruri (2005), “Crafting Migrant Women's Citizenship in Japan: Taking 'Family' as a Vantage Point", International Journal of Japanese Sociology, núm. 14, pp. 52-67.

Jones, H. J. (1975), "Shiryô Oyatoi Gaikokujin’ by UnEsco Higashi Ajia Bunka Kenkyu Senta”, Monumenta Nipponica, 30(4), pp. 465-468.

Joppke, Christian (1999), "How immigration is changing citizenship: A comparative view", Ethnic and Racial Studies, 22(4), julio, pp. 629-652.

Kajita, Takamichi (1994), Gaikokujin rodôsha to nihonjin, Tokio: Nippon Hoso Kyokai.

- - (2001), "Mittsu no 'shitizunshippu' - 'mittsu no gêto' ron ni yoru seiri”, en NIRA Citizenship Kenkyukai (ed.), Tabunkashakai no Sentaku: Shitizunshippu no Shiten kara, Tokio: Nihon Hyoronsha.

Kajita, Takamichi, Tanno, Kiyoto, y Higuchi, Naoto (2006), Kao no mienai teijûka. Nikkei burajirujin to kokka, shijô, imin nettowaaku, Nagoya: Nagoya Daigaku Shuppankai.

Kashiwazaki, Chikako (2000), "Citizenship in Japan: Legal practice and contemporary development”, en Aleinikoff, T. A., y Klusmeyer, D. (eds.), From Migrant to Citizens: Membership in a Changing World, Washington, DC: Carnegie Endowment for International Peace.

Kibe, Takashi (2006), "Differentiated Citizenship and Ethnocultural Groups: A Japanese Case”, Citizenship Studies, 10(4), septiembre, pp. 413-430.

Kim, Bumsoo (2006), "From Exclusion to Inclusion? The Legal Treatment of 'Foreigners' in Contemporary Japan", Immigrants \& Minorities, 24(1), marzo, pp. 51-73.

Komai, Hiroshi (1997), "Hajime ni uchinaru kokusaika ni yoru tabunka kyôsei shakai no kôchiku”, en Komai, Hiroshi, y Watado, Ichiro (eds.), Jichitai no gaikokujin seisaku - uchinaru kokusaika e no torikumi, Tokio: Akashi Shoten.

_- (2001), Foreign Migrants in Contemporary Japan, Melbourne: Trans Pacific Press.

_- (2006), Gurobaruka jidaino nihongata tabunka kyôsei shakai, Tokio: Akashi Shoten.

Kymlicka, Will (1989), Liberalism, Community and Culture, Oxford: Clarendon Press.

(1995), Multicultural Citizenship: A Liberal Theory of Minority Rights, Oxford: Oxford University Press. 
Lee, Michelle Anne (2003), "Multiculturalism as nationalism: A discussion of nationalism in pluralistic nations", Canadian Review of Studies in $\mathrm{Na}$ tionalism, núm. Xxx, pp. 103-123.

Linger, Daniel Touro (2001), No one Home: Brazilian Selves Remade in Japan, Stanford, California: Stanford University Press.

Marshall, Thomas H., y Bottomore, Tom (1992), Citizenship and Social Class, Londres: Pluto Press, pp. 1-84.

McCormack, Gavan (2001), "Kokusaika: Impediments in Japan's deep structure”, en Denoon, Donald, et al. (eds.), Multicultural Japan: Paleolithic to Postmodern, Nueva York: Cambridge University Press.

Méndez Vázquez, Marcela Inés (1998), Foreign Bodies: Migrant Latina Sex Workers in Contemporary Japan, Masters' thesis, Tokio: Sophia University. (2008), "South American nikkeijin Acquisition of Local Citizenship: The Case of the Japanese-Peruvian Association -AJAPE", en Mita Chiyoko, et al. (eds.), Sociedade Japonesa e Migrantes brasileiros: Novos Caminhos na Formação de uma Rede de Pesquisadores, Tokio: Sophia University-Center for Luso-Brazilian Studies.

Milly, Deborah J. (2006), “Policy Advocacy for Foreign Residentes in Japan”, en Tsuda, Takeyuki (ed.), Local Citizenship in Recent Countries of Immigration: Japan in Comparative Perspective, Maryland: Lexington Books.

Oguma, Eiji (1998), "Nihonjin" no kyôkai. Okinawa, Ainu, Taiwan, Chôsen, shokuminchi shihai kara fukki undô made (The boundaries of the Japanese). Tokio: Shinyosha.

- - (2002), A Genealogy of Japanese Self-Images, Rosanna/Portland: Trans Pacific Press.

Okamoto, Masataka (2005), Nihon no minzoku sabetsu jinshu sabetsu teppai jôyaku, Tokio: Akashi Shoten.

Parekh, Bhikhu (2000), Rethinking Multiculturalism: Cultural Diversity and Political Theory, Cambridge, Mass.: Harvard University Press.

Portes, Alejandro, y Rumbaut, Rubén G. (2001), Legacies. The Story of the Immigrant Second Generation, Berkeley, CA: University of California Press. Roth, Joshua Hotaka (2002), Brokered Homeland: Japanese Brazilian Migrants in Japan, Ithaca, NY: Cornell University Press.

Sakai, Alberto (2006), "Dekasegi no jûgonen. Nikkeisei wo ikiru michi”, en Sakurai, Atsushi (ed.), Sengo sesô no keikenshi, Tokio: Serika Shobo.

Sakanaka, Hidenori, y Asakawa, Akihiro (2007), Imin Kokka Nippon: 1000-man nin no Imin ga Nippon wo Sukû, Tokio: Nippon Kajo Shuppan. 
Saruhashi, Junko (2007), "Kyôdô sagyô to shite no gengo sâbisu. Kawasakishi no jirei kara”, en Kawahara, Toshiaki, y Noyama, Hiroshi (eds.), Gaikokujin jûmin e no gengo sâbisu: chîki shakai -jichitai wa tagengo shakai o dô mukaeru, Tokio: Akashi Shoten.

Shipper, Apichai (2002), "The political construction of foreign workers in Japan”, Critical Asian Studies, núm. 34, pp. 41-68.

- - (2008), Fighting for Foreigners: Immigrants and its Impact on Japanese Democracy, Ithaca, NY: Cornell University Press.

Siddle, Richard (1996), Race, resistance, and the Ainu of Japan. Nueva York: Routledge.

Tai, Eika (2004), "Korean Japanese. A New Identity Option for Resident Koreans in Japan", Critical Asian Studies, 36(3), pp. pp. 355-382.

Takahashi, Etsuko (2007a), Camino para la educación superior, presentación en la conferencia: "Dai Sankai Kyôiku Fôramu 'Riidashippu, Komyûniti, Tôgo"', organizada por Nihon-Peru Kyôsei Kyôkai, Machida Citizens Hall, 11 de noviembre.

(2007b), Supeingoken kara mita chiikirentai nettowaaku, presentación en la conferencia: "Dai Ikkai Zenkoku Fôramu 'Tabunka Kyôdô jissen kenkyû"', organizada por el Center for Multilingual - Multicultural Education and Research, Tokyo University of Foreign Languages, Fuchu Campus, 3 de diciembre.

Takenaka, Ayumi (2000), Ethnic community in motion: Japanese-Peruvians in Peru, Japan, and the United States, tesis doctoral, Nueva York: Columbia University.

-_ (2009), "Ethnic Hierarchy and Its Impact on Ethnic Identities: A Comparative Analysis of Peruvian and Brazilian Return Migrants in Japan", en Tsuda, Takeyuki, Diasporic Homecomings: Ethnic Return Migration in Comparative Perspective, Stanford, CA: Stanford University Press.

Tegtmeyer Pak, Katherine (2001), “Towards Local Citizenship: Japanese Cities Respond to International Migration", Center for Comparative Immigration Studies. Working Papers. http://repositories.cdlib.org/ccis/papers/wrkg30 Tsuda, Takeyuki (2003), Strangers in the Ethnic Homeland: Japanese Brazilian Return Migration, Nueva York: Columbia University Press.

- - (ed.) (2006), Local Citizenship in Recent Countries of Immigration: Japan in Comparative Perspective, Maryland: Lexington Books.

-_ (ed.) (2009), Diasporic Homecomings: Ethnic Return Migration in Comparative Perspective, Stanford, CA: Stanford University Press. 
Williams, Melissa S. (1998), Voice, Trust, and Memory. Marginalized Groups and the Failings of Liberal Representation, Princeton: Princeton University Press.

Yamanaka, Keiko (2003), "Feminization of Japanese Brazilian labor migration to Japan”, en Lesser, Jeffrey (ed.), Searching for home abroad: Japanese Brazilians and transnationalism, Durham: Duke University Press.

- - (2004), "Imin tôgô katei ni okeru josei no yakuwari to igi: Nikkei burajirujin hahaoya gurûpu no kyôiku shien jirei no kôsatsu”, en Ito, Ruri (ed.), Gendai Nihon shakai ni okeru kokusai imin to jendâ kankei no saihen ni kansuru kenkyû, Tokio: Kagaku Kenkyuhi Hojokin Kenkyu Seika Hokokusho/Ochanomizu University-Institute for Género Studies.

- - (2006), "Immigrant Incorporation and Women's Community Activities in Japan: Local NGOs and Public Education for Immigrant Children", en Tsuda, Takeyuki (ed.), Local Citizenship in Recent Countries of Immigration: Japan in Comparative Perspective, Maryland: Lexington Books.

Yamawaki, Keizo (2005), “Nisengonen wa tabunka kyôusei gannen?”, Jichitai Kokusaika Forum. http://www.clair.or.jp/j/forum/forum/culture/187/ index.html

Yorimitsu, Masatoshi (ed.) (2006), Nihon no imin seisaku wo kangaeru. Jinkô genshô shakai no kadai, Tokio: Akashi Shoten.

Young, Iris Marion (1986) "Deferring Group Representation", en Kymlicka, Will, y Shapiro, Ian (eds.), Nomos: Group Rights, New York: Nueva York University Press, pp. 349-376.

- - (1989), "Polity and group difference: A critique of the ideal of universal Citizenship", Ethics, núm. 99, pp. 250-274. http://www.clair.or.jp/j/ forum/forum/culture/187/index.html

Yuval-Davis, Nira (1997), "Women, Citizenship and Difference", Feminist Review, núm. 57, otoño, pp. 4-27. 